\title{
Hemoglobina de reticulocito y su importancia en el diagnóstico temprano de anemia ferropénica
}

\author{
Reticulocyte hemoglobin and its importance in early diagnosis of iron deficiency anemia
}

Yusselfy Márquez-Benítez' orcid.org/0000-0002-7677-6329

Shirley Gigiola Cruz-Rubio'* orcid.org/0000-0003-2964-0602

Diana Marcela Vargas-Acevedo' orcid.org/0000-0003-2237-5973

1 Grupo de Investigación Bacteriología y Laboratorio Clínico (GRIBAC), Universidad de Boyacá. Boyacá, Colombia.

\section{Resumen}

Introducción: El contenido de hemoglobina de reticulocitos (CHr), es un parámetro en la biometría hematológica automatizada que proporciona información sobre el contenido de hierro, por ello se ha utilizado como un marcador de la biodisponibilidad del hierro en la eritropoyesis, permite su detección en una etapa temprana de la anemia ferropénica y otras patologías como inflamación crónica, enfermedad renal crónica; además realizar monitoreo de terapias con eritropoyetina y hierro. Objetivo: Exponer la aplicabilidad de la $\mathrm{CHr}$ como un parámetro en el diagnóstico precoz de la anemia por deficiencia de hierro, así como su medición e interpretación. Materiales y métodos: Se realizó la revisión de artículos científicos en inglés y español en las bases de datos PubMed, ScienceDirect, LILACS y Medline, usando descriptores validados en Medical Subject Headings (MeSH), considerando periodo de publicabilidad del $80 \%$ inferior a 5 años. Resultados: Se describe la importancia, aplicabilidad, determinación e interpretación de este parámetro como biomarcador específico hemático temprano en el diagnóstico de deficiencia de hierro antes de presentarse cambios morfológicos eritroides. Conclusiones: La CHr es un parámetro de gran utilidad en el diagnóstico temprano de anemia ferropénica y otras patologías como deficiencia funcional de hierro, estados de inflamación crónica y enfermedad renal crónica.

Palabras clave: Anemia; deficiencia de hierro; reticulocitos; hierro; biomarcadores. (Fuente: DeCS, Bireme).

\begin{abstract}
Introduction: The reticulocyte hemoglobin content $(\mathrm{CHr})$ is a parameter in automated hematological biometrics, which can provide information on the iron content. So it has been used as a marker of the bioavailability of iron in the erythropoiesis, it allows its detection at an early stage of iron deficiency anemia and other pathologies such as chronic inflammation, chronic kidney disease; in addition to monitoring therapy with erythropoietin and iron. Objective: To expose the applicability of $\mathrm{CHr}$ as a parameter in the early diagnosis of iron deficiency anemia, as well as its measurement and interpretation. Materials and methods: The review of scientific articles in English and Spanish was carried out in the PubMed, ScienceDirect, LILACS and Medline databases, using descriptors validated in Medical Subject Headings (MeSH), considering the publication period of $80 \%$ less than 5 years. Results: The importance, applicability, determination and interpretation of this parameter is described as an early specific biomarker in the blood in the diagnosis of iron deficiency before presenting morphological changes occurring during terminal erythroid differentiation. Conclusions: $\mathrm{CHr}$ is a very useful parameter in the early diagnosis of iron deficiency anemia and other pathologies such as functional deficiency, chronic inflammation states and chronic renal disease.
\end{abstract}


Key words: Anemia; iron deficiency; reticulocytes; iron; biomarkers. (Source: DeCS, Bireme).

\section{Introducción}

A nivel mundial se ha considerado que la anemia por deficiencia de hierro (DH) es una de las carencias nutricionales más frecuentes, los cálculos más recientes de la Organización Mundial de la Salud (OMS) sugieren que la anemia afecta a alrededor de 800 millones de niños y mujeres. De hecho, 528,7 millones de mujeres y 273,2 millones de niños menores de 5 años eran anémicos en 2011, por las etapas de crecimiento, mujeres en edad fértil debido a las pérdidas de sangre en las menstruaciones, embarazadas debido al aumento de los requerimientos de hierro como consecuencia del rápido crecimiento de la placenta y del feto, en los ancianos debido a la ingesta de dietas inadecuadas o por la aparición de enfermedades que provocan esta condición(1).

El diagnóstico de laboratorio de la anemia por deficiencia de hierro, exige en primer lugar la confirmación de la depleción de las reservas de hierro, para que sea posible establecer la etiología de la enfermedad. La aparición de la anemia es el último estadio de la deficiencia de hierro, donde la producción de hemoglobina está comprometida y consecuentemente la sobrevida eritrocitaria debido a la carencia de hierro, en la mayoría de los casos, ésta fase es diagnosticada por la presencia de microcitosis e hipocromía en el extendido de sangre periférica. La evidencia bioquímica de agotamiento de las reservas de hierro esta mediado por marcadores bioquímicos clásicos tales como los niveles de hierro sérico, transferrina y ferritina ${ }^{(2,3)}$.

En los últimos años se han producido importantes avances en la detección precoz del déficit de hierro, con la introducción de nuevos parámetros hematológicos y bioquímicos del metabolismo férrico(4). Los analizadores hematológicos proporcionan el recuento de reticulocitos automatizado, representando numerosas ventajas sobre el método manual, proporcionando parámetros adicionales como contenido de hemoglobina y fracción de reticulocitos inmaduros(5). Algunos autores han señalado que el contenido de hemoglobina reticulocitaria $(\mathrm{CHr})$ es el marcador más directo de una adecuada síntesis de hemoglobina ${ }^{(6,7)}$, estos índices reticulocitarios proveen una evaluación en tiempo real de la actividad de la medula ósea, y reflejan el balance entre el hierro y la eritropoyesis ${ }^{(8,9)}$.

Es por esto que, a través de una revisión de literatura exhaustiva, se pretende examinar y mostrar la aplicabilidad del $\mathrm{CHr}$ como un parámetro en el diagnóstico precoz de la anemia por deficiencia de hierro; así mismo se hace una revisión sobre su medición dentro del laboratorio e interpretación.

\section{Materiales y métodos}

Se tuvo en cuenta criterios de inclusión para obtener un documento de calidad, basados en el objetivo de la revisión. Se realizó una búsqueda electrónica en las bases de datos PubMed, ScienceDirect, LILACS y Medline, con términos claves como: anemia, deficiencia de hierro, hemoglobina reticulocitaria, ferropenia, biomarcadores y la combinación de ellos que incluyeron artículos de revisión y productos de investigación; además de la utilización de libros electrónicos que apoyaron la fundamentación teórica. Se tomaron en cuenta artículos completos en idioma inglés y español, con un periodo de publicabilidad del $80 \%$ no mayor a 5 años.

\section{Deficiencia De Hierro}

La DH se define como una condición en la que hay una reducción de hierro corporal total, con agotamiento de las reservas y algún grado de deficiencia de hierro en los tejidos. El desarrollo de la anemia por deficiencia de hierro es

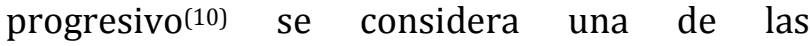
deficiencias nutricionales más comunes y la principal causa de anemia principalmente en mujeres y niños(1,11). 
La falta de hierro puede ser: absoluta cuando hay una reducción real de hierro corporal total del organismo; o funcional cuando a pesar de existir hierro, este no se aprovecha adecuadamente para la eritropoyesis. La distribución de hierro tiene su propia dinámica y puede ocupar diferentes compartimientos que están interligados, esto puede conllevar a un punto que concurre en la disminución de la cantidad de hemoglobina presente en la sangre ${ }^{(11,12)}$. La hemoglobina se encuentra en los glóbulos rojos desde el normoblasto policromático y en los reticulocitos se denomina hemoglobina reticulocitaria(13,14).
Desde el laboratorio clínico existen diversos parámetros que evalúan el estado de hierro funcional (Tabla 1). Existen pruebas de laboratorio para confirmar el agotamiento de hierro, que son clasificadas de acuerdo con su actividad específica en ensayos directos e indirectos. La evidencia directa no se utiliza de forma rutinaria a pesar de su mayor especificidad, ya que se considera invasiva e incómoda para el paciente(15-17). Por el contrario, las evidencias indirectas son más accesibles a los pacientes y ampliamente utilizadas en la rutina de laboratorio(18).

Tabla 1. Estadios de anemia por deficiencia de hierro.

\begin{tabular}{|c|c|c|}
\hline Marcadores de almacenamiento & Niveles de normalidad & Deficiencia de hierro \\
\hline Hierro sérico(19) & $\begin{array}{l}\text { Hombres: } 50-160 \mathrm{ug} / \mathrm{dL} \\
\text { Mujeres: } 60-150\end{array}$ & Disminuido \\
\hline Almacenamiento de médula ósea(20) & $\begin{array}{l}\text { En condiciones normales se observan } \\
\text { sideroblastos en cuyo interior aparecen } \\
\text { de } 1 \text { a } 4 \text { gránulos en el citoplasma. }\end{array}$ & Disminuido \\
\hline $\begin{array}{l}\text { Capacidad total de fijación de } \\
\text { hierro(21) }^{(2)}\end{array}$ & $205-400 \mathrm{ug} / \mathrm{dL}$ & Aumentada \\
\hline Protoporfirina zinc eritrocitaria(19) & $0-70 \mu \mathrm{g} / \mathrm{dL}$ & Aumentada \\
\hline Ferritina sérica(19) & $\begin{array}{l}\text { Hombres: } 15-400 \mathrm{ng} / \mathrm{mL} \\
\text { Mujeres: } 10-106 \mathrm{ng} / \mathrm{mL} \\
\text { Niños: } 10-106 \mathrm{ng} / \mathrm{mL}\end{array}$ & $\begin{array}{l}\text { Aumentada } \\
\text { *Por ser una proteína de fase aguda, los } \\
\text { niveles aumentan en estados de } \\
\text { inflamación aguda o crónica, neoplasias, } \\
\text { hepatopatías. }\end{array}$ \\
\hline$\%$ de saturación de la transferrina(19) & $\begin{array}{l}\text { Límite inferior } \\
6 \text { meses a } 6 \text { años: } 9 \% \\
7 \text { años- } 12 \text { años: } 11 \% \\
\text { Adultos: } 16 \%\end{array}$ & Disminuida \\
\hline Hepcidina(22) & $\begin{array}{l}\text { hombres: 2,1-15,1 nM } \\
\text { mujeres: 1,6-15,6 nM }\end{array}$ & Aumentado \\
\hline $\begin{array}{l}\text { Receptor soluble de la } \\
\text { transferrina( }(23,24)\end{array}$ & $8.7-28.1 \mathrm{nmol} / \mathrm{L}$ & Aumentada \\
\hline Hemoglobina reticulocitaria(25) & $>26 \mathrm{pg}$ & Disminuida $<26$ pg. \\
\hline Morfología eritroide(20) & Normocítica/normocrómica & Microcíticos/hipocrómicos \\
\hline
\end{tabular}

Los marcadores bioquímicos que evalúan el suministro de hierro a la médula ósea son indicadores indirectos entre el hierro y la eritropoyesis; entre las pruebas indirectas que pueden ser utilizadas en la investigación de los trastornos de hierro, se destacan: el contenido de hierro en plasma, la ferritina sérica, transferrina, contenido de hemoglobina plasmática, índices de eritrocitos, concentración de hemoglobina corpuscular media (CHCM), Amplitud de la Distribución Eritrocitaria o RDW, por su nombre en inglés: Red blood cell Distribution Width, receptores solubles de transferrina (sTfR), y parámetros de reticulocitos(17,8). El diagnóstico de la deficiencia de hierro se basa en la presencia de anemia y morfología de eritrocitos (hipocromía y microcitosis) junto con la dosificación sérica disminuida de la ferritina(26). 


\section{Marcadores bioquímicos para el diagnóstico de la eritropoyesis deficiente en hierro}

La eritropoyesis deficiente en hierro básicamente se sospecha por la clínica, los factores epidemiológicos y los hallazgos del hemograma; no obstante, en todos los casos se debe confirmar a través de las pruebas de laboratorio conocidas como biomarcadores. Con el paso de los años se han incorporado al laboratorio diferentes biomarcadores para el diagnóstico y seguimiento de la eritropoyesis deficiente en hierro ${ }^{(23,27,28)}$ y durante los últimos años la hemoglobina reticulocitaria se ha perfilado como un parámetro de gran utilidad clínica, teniendo en cuenta que se puede incorporar dentro de los autoanalizadores de hematología y puede ser parte de los marcadores de rutina para el diagnóstico y seguimiento de la ferropenia según lo muestran diferentes investigaciones(29-31).

\subsection{Hierro medular}

Si bien el estándar de oro para definir la deficiencia absoluta de hierro es la búsqueda de depósitos en la medula ósea mediante la coloración de azul de Prusia, en la actualidad ésta prueba ha perdido vigencia y ha sido remplazada por el uso de biomarcadores; debido a que esta tinción requiere un aspirado de medula ósea(17), procedimiento que sólo se puede realizar en centros especializados en hematología; además, la coloración del hierro medular es un procedimiento complicado, que requiere la obtención mínima de siete fragmentos óseos, no se encuentra debidamente estandarizado y es altamente dependiente del observador(32).

\subsection{Ferritina sérica}

Es la prueba más requerida en el estudio del metabolismo de hierro, la ferritina es una proteína soluble que almacena hierro que corresponde al excedente que no fue utilizado en el metabolismo normal. Los niveles séricos de la ferritina son directamente proporcionales a la cantidad de hierro de las reservas tisulares, de esta manera son un reflejo del estado de las reservas de hierro, también conocidas como hierro de depósito, por lo que cada microgramo de ferritina sérica equivale a $10 \mathrm{mg}$ de hierro de reserva(33). Cuando las reservas de hierro están normales se espera que los niveles de la ferritina sérica se encuentren en el rango de variabilidad biológica, siempre y cuando no se presente concomitante con procesos inflamatorios e infecciosos crónicos, ya que por ser esta un reactante de fase aguda la interpretación clínica se ve alterada; en el caso de la deficiencia de hierro los niveles de ferritina sérica usualmente se encuentran disminuidos según el estadio, por debajo de $12 \mathrm{ng} / \mathrm{L}$ (estadio ll y lll) o entre 12 ng/L y 30 ng/L (estadio l)(11).

\subsection{Hierro sérico}

Corresponde a la forma de hierro que viaja unida a la transferrina, por lo que para su medición analítica se induce la liberación de la transferrina y posteriormente se determina por métodos colorimétricos, el valor de referencia para el hierro sérico es de $60 \mathrm{mg} / \mathrm{dl}$ a $170 \mathrm{mg} / \mathrm{dl}\left({ }^{34)}\right.$. La hipoferremia se presenta en la anemia asociada con inflamación, aquí el hierro de depósito puede ser normal o aumentado, pero se da la reducción de la eritropoyesis y acorta la vida media de los eritrocitos por acción de la hepcidina $(35,36)$, es por ello que el hierro sérico no se puede usar como único biomarcador para el diagnóstico de la deficiencia de hierro, en especial cuando el paciente presenta comorbilidades asociadas una anemia crónica.

Similar a lo que sucede con la ferritina sérica, los niveles de hierro están sujetos a las variaciones circadianas y al tipo de alimentación del paciente el día anterior a la toma de la muestra, así como a la amplia variabilidad biológica del analito(37).

\subsection{Capacidad total de la fijación de hierro}

Es una medida indirecta de la transferrina sérica, proteína a la cual se une el hierro para su transporte en plasma hacia los tejidos(19). Este marcador se considera un reflejo de la cantidad total de hierro circulante en el plasma, incluyendo el hierro que se puede unir a la transferrina. En condiciones normales, la capacidad total de fijación del hierro oscila entre $240 \mathrm{mg} / \mathrm{dl}$ a $450 \mathrm{mg} / \mathrm{dl}$. En la anemia por deficiencia de hierro la capacidad total de fijación del hierro se encuentra elevada(19,38). 


\subsection{Saturación de transferrina}

Corresponde a la relación entre el hierro sérico y la capacidad total de fijación del hierro, expresada en porcentaje. La interpretación del resultado se debe tener en cuenta las variaciones relacionadas con las mediciones de estos dos analitos que intervienen en su cálculo. Bajo condiciones normales, los valores de la saturación de la transferrina oscilan entre $20 \%$ y $50 \%$. En la deficiencia absoluta de hierro los niveles de hierro sérico están disminuidos y la capacidad total de fijación del hierro aumentada, lo que usualmente da como resultado una saturación de la transferrina baja, inferior a $20 \%{ }^{(39)}$.

\subsection{Receptor soluble de transferrina}

Es uno de los biomarcadores de mayor interés en los últimos años. La transferrina tiene un receptor específico de membrana, el receptor de la transferrina tipo 1 y el tipo 2, la síntesis del receptor de transferrina se ha observado que depende directamente de los niveles de hierro en el organismo. De esta forma, si los niveles de hierro bajan se inducen la síntesis del receptor y cuando los niveles de hierro son los adecuados se previene su producción. Este mecanismo de regulación en conjunto con el conocimiento de que el receptor soluble de la transferrina refleja la entrega de hierro a los eritroblastos, es decir la tasa de eritropoyesis, ha hecho que su medición cobre especial interés en los últimos años, además de que permite la diferenciación entre la anemia por deficiencia de hierro (niveles elevados del receptor) y la anemia por enfermedad crónica (niveles normales o disminuidos)(40,41).

\subsection{Hepcidina}

Es un péptido de origen hepático con propiedades antimicrobianas, que actúa como regulador de la absorción y cinética del hierro en el organismo(42). En el 2000 se describió la hepcidina por primera vez como un péptido antimicrobiano y un año más tarde se relacionó con el metabolismo del hierro, junto a otros genes y proteínas, bajo su nombre actual(42.43). Aunque inicialmente se le reconoció a la hepcidina únicamente una actividad antimicrobiana in vitro, recientemente se le ha dado un valor de primer orden en la homeostasis del hierro, ya que la hepcidina por medio de la internalización y degradación de la proteína exportadora de hierro (ferroportina-l), inhibe la absorción de hierro en el ámbito intestinal, bloquea el trasporte de hierro a través de la placenta e induce el secuestro del hierro en macrófagos y hepatocitos(42). La síntesis de hepcidina esta modulada tanto por los requerimientos de hierro del organismo como por estados inflamatorios, infecciosos y sobre carga de hierro no genético, como en pacientes politransfundidos y disminuye en la anemia ferropénica(43).

En general, no existe una prueba de rutina utilizada en el laboratorio, con una sensibilidad y especificidad suficiente para ser seleccionado como marcador definitivo de la deficiencia de hierro, pero normalmente se emplean pruebas bioquímicas mencionadas anteriormente $(37,44)$. Los parámetros de reticulocitos se presentan como una buena alternativa para el diagnóstico de deficiencia de hierro, debido a que proporcionan información que corresponde a contenido de hemoglobina en los eritrocitos que recién salen a circulación y por lo tanto un indicador temprano de la biodisponibilidad de hierro(45,46). Este contenido proporciona una evaluación de la actividad en la maduración de la línea eritroide, que refleja el equilibrio entre el hierro y la eritropoyesis.

\section{Hemoglobina reticulocitaria como marcador de eritropoyesis deficiente de hierro dentro del hemograma automatizado}

A partir de la introducción de los autoanalizadores de hematología se incluyeron nuevos parámetros al hemograma, que permiten un acercamiento a la identificación de la etiología de la anemia; el $\mathrm{CHr}$ se convirtió en una herramienta eficaz para el diagnóstico de la anemia por deficiencia de hierro ${ }^{(4)}$.

El CHr o la concentración de hemoglobina corpuscular media de reticulocitos (HCMR), es considerada un nuevo parámetro eritrocitario de gran importancia clínica, que se encuentra disponible en los auto analizadores de hematología de última generación la cual 
determina la cantidad de hemoglobina presente en los reticulocitos en picogramos como unidad de peso(47,48). El CHr, corresponde a la hemoglobinización de los reticulocitos de las últimas 48 a 72 horas. CHr es a los reticulocitos lo que la hemoglobina corpuscular media es a los eritrocitos, en la medida en que ambos parámetros miden la cantidad de hemoglobina presente en cada célula(49), esta prueba sólo está disponible en los hemogramas tipo VI como se muestra en la Tabla 2.

Tabla 2. Tipos de hemogramas de acuerdo con la tecnologia y parámetros evaluados

\begin{tabular}{|c|c|c|c|c|c|c|}
\hline Parámetro/Tipo & I & II & III & IV & $\mathbf{V}$ & VI \\
\hline Hemoglobina(50-52) $^{(52}$ & $\begin{array}{l}\text { Determinación } \\
\text { directa }\end{array}$ & $\begin{array}{l}\text { Determinación } \\
\text { directa }\end{array}$ & $\begin{array}{l}\text { Determinación } \\
\text { directa }\end{array}$ & $\begin{array}{l}\text { Determinación } \\
\text { directa }\end{array}$ & $\begin{array}{l}\text { Determinación } \\
\text { directa }\end{array}$ & $\begin{array}{l}\text { Determinación } \\
\text { directa }\end{array}$ \\
\hline Hematocrito(50-52) $^{(2)}$ & $\begin{array}{l}\text { Determinación } \\
\text { directa }\end{array}$ & $\begin{array}{l}\text { Determinación } \\
\text { directa }\end{array}$ & $\begin{array}{l}\text { Determinación } \\
\text { directa }\end{array}$ & $\begin{array}{l}\text { Determinación } \\
\text { directa }\end{array}$ & $\begin{array}{l}\text { Determinación } \\
\text { directa }\end{array}$ & $\begin{array}{l}\text { Determinación } \\
\text { directa }\end{array}$ \\
\hline $\begin{array}{l}\text { Recuento de } \\
\text { eritrocitos }(50-52)\end{array}$ & $\begin{array}{l}\text { Determinación } \\
\text { directa }\end{array}$ & $\begin{array}{l}\text { Determinación } \\
\text { directa }\end{array}$ & $\begin{array}{l}\text { Determinación } \\
\text { directa }\end{array}$ & $\begin{array}{l}\text { Determinación } \\
\text { directa }\end{array}$ & $\begin{array}{l}\text { Determinación } \\
\text { directa }\end{array}$ & $\begin{array}{l}\text { Determinación } \\
\text { directa }\end{array}$ \\
\hline $\begin{array}{l}\text { Índices } \\
\text { eritrocitarios }{ }^{(50-52)}\end{array}$ & $\begin{array}{l}\text { Determinación } \\
\text { por Cálculo }\end{array}$ & $\begin{array}{l}\text { Determinación } \\
\text { por Cálculo }\end{array}$ & $\begin{array}{l}\text { Determinación } \\
\text { por Cálculo } \\
\text { Volumen } \\
\text { Corpuscular } \\
\text { Medio: } \\
\text { Determinación } \\
\text { directa }\end{array}$ & $\begin{array}{l}\text { Determinación } \\
\text { por Cálculo } \\
\text { Volumen } \\
\text { Corpuscular } \\
\text { Medio: } \\
\text { Determinación } \\
\text { directa }\end{array}$ & $\begin{array}{l}\text { Determinación } \\
\text { por Cálculo } \\
\text { Volumen } \\
\text { Corpuscular } \\
\text { Medio: } \\
\text { Determinación } \\
\text { directa }\end{array}$ & $\begin{array}{l}\text { Determinación por } \\
\text { Cálculo } \\
\text { Volumen } \\
\text { Corpuscular Medio: } \\
\text { Determinación } \\
\text { directa }\end{array}$ \\
\hline $\begin{array}{l}\text { Recuento total de } \\
\text { leucocitos }^{(50-52)}\end{array}$ & Manual & Manual & Semiautomatizado & Electrónico & Electrónico & Electrónico \\
\hline $\begin{array}{l}\text { Recuento de } \\
\text { leucocitos } \\
\text { diferencial(50-52) }\end{array}$ & Manual & Manual & Semiautomatizado & Electrónico & Electrónico & Electrónico \\
\hline $\begin{array}{l}\text { Morfología } \\
\text { celular(50-52) }\end{array}$ & Manual & Manual & Manual & Electrónico & $\begin{array}{l}\text { Electrónico/ } \\
\text { manual }\end{array}$ & $\begin{array}{l}\text { Electrónico/ } \\
\text { manual }\end{array}$ \\
\hline $\begin{array}{l}\text { Recuento de } \\
\text { plaquetas }^{(50-52)}\end{array}$ & & Manual & Semiautomatizado & Electrónico & Electrónico & $\begin{array}{l}\text { Electrónico } \\
\text { Plaquetas } \\
\text { reticuladas }\end{array}$ \\
\hline $\begin{array}{l}\text { Índices } \\
\text { plaquetarios }^{(52)}\end{array}$ & & & & & Electrónico & Electrónico \\
\hline $\begin{array}{l}\text { Recuento } \\
\text { de reticulocitos, } \\
\text { índices } \\
\text { reticulocitarios, } \\
\text { hemoglobina } \\
\left.\text { reticulocitaria( }{ }^{52}\right)\end{array}$ & & & & & & Electrónico \\
\hline
\end{tabular}

\subsection{Historia}

En 1994 Brugnara et al.(53), fueron unos de los primeros investigadores en utilizar el contenido de $\mathrm{CHr}$ para monitorización de la terapia con hierro, en un estudio con 10 mujeres que presentaban anemia por deficiencia de hierro; los resultados mostraron que el recuento de reticulocitos del grupo en promedio pasó de 65.150 a 130.450 por UL, mientras que la $\mathrm{CHr}$ aumentó de 18 pg a 23,4 pg durante las dos primeras semanas de tratamiento. En el mismo estudio, se comparó el aumento de $1 \mathrm{~g} / \mathrm{dl}$ de hemoglobina (producido sólo 28 días después del inicio del tratamiento), con la hemoglobina reticulocitaria, observando que ésta aumento en primera medida en comparación con la hemoglobina total plasmática.

En 1997 Fishbane et al.(54), evaluaron el contenido de $\mathrm{CHr}$ sobre el estado del metabolismo del hierro en 164 pacientes en tratamiento con hemodiálisis. Con $100 \%$ de sensibilidad y $80 \%$ de especificidad, los resultados reportaron que el CHr es el parámetro de evaluación principal de la biodisponibilidad del hierro en la médula ósea, el CHr también puede ser útil para identificar a los pacientes que no responden a tratamiento oral con hierro cuando el CHr sigue siendo baja incluso después del inicio de su consumo, en estos casos se indica terapia intravenosa. 
En 1994 Brugnara et al.(55), realizaron un estudio en el Children's Hospital y The Brigham and Women's Hospital de Boston; de los índices de la maduración de eritrocitos y de reticulocitos en 10 mujeres adultas con deficiencia de hierro antes de la terapia y 1 o 2 semanas durante la terapia de reemplazo de hierro por vía oral (324 mg de gluconato ferroso dos veces al día), para la medición se utilizó el analizador de Oxazina por citometría de Flujo 750- stained reticulocytes with the Miles $H^{*} 3$. En todos los pacientes, la terapia oral con hierro fue asociada con un incremento significativo del conteo absoluto de reticulocitos y el contenido de $\mathrm{CHr}$ de $18 \pm 3,2 \mathrm{pg}$ a 23,4 $\pm 3,1$ pg. No hubo cambios en los índices de glóbulos rojos y/o cambios significantes en la hemoglobina corpuscular media. Este aumento constante en el conteo absoluto de reticulocitos y el contenido de CHr sugirió que estas medidas de respuesta al tratamiento pueden ser confiables poco después de iniciar el tratamiento. El $\mathrm{CHr}$ puede también ser usado para identificar pacientes que no responden a la terapia oral con Hierro. Estos datos preliminares sugirieron que los índices reticulocitarios pueden permitir una evaluación en tiempo real de la eritropoyesis con deficiencia de hierro y de la eficacia de la terapia de reemplazo de hierro.

En 2002 Mast et al.(49), reportaron un estudio donde evaluaron la utilidad clínica del contenido de $\mathrm{CHr}$ para el diagnóstico de deficiencia de hierro a 78 pacientes que después de firmar el consentimiento informado fueron sometidos a examen de medula ósea, en el mismo día se obtuvo una muestra de sangre periférica tomada en $K_{3}$ EDTA de cada paciente, para realizarle los análisis de rutina $\mathrm{y}$ análisis reticulocitarios incluyendo el $\mathrm{CHr}$, utilizando el analizador hematológico Advia 120. De los 78 pacientes, el $36 \%$ presentaron deficiencia de hierro, donde el rango de $\mathrm{CHr}$ fue de 21,0 a 38,6 pg mientras que en pacientes sin deficiencia de hierro el rango de $\mathrm{CHr}$ fue de 22,8 a 43,7 pg. La habilidad del $\mathrm{CHr}$ de predecir el almacenamiento de hierro en médula ósea fue comparada con el punto de corte de la ferritina, el porcentaje de saturación de transferrina y el tamaño promedio de los glóbulos rojos o Volumen Corpuscular Medio (VCM) por la curva de análisis Roc y fueron presentados de acuerdo a su sensibilidad 52,4\%, $65 \%$ y $31,8 \%$ respectivamente y especificidad $92,9 \%, \quad 70,3 \% \quad$ y $93,3 \%$ respectivamente; mostrando que el CHr tuvo en general la mayor sensibilidad y especificidad del resto de análisis de sangre periférica, prediciendo la ausencia de depósitos de hierro en la médula ósea.

En la actualidad diversos estudios comparan la sensibilidad y especificidad del índice reticulocitario con diferentes biomarcadores en la evaluación de los estados del hierro, ya sea en población de gestantes, infantes, donantes e inclusive en pacientes con diálisis en donde mencionan que la hepcidina ha presentado correlación con otros marcadores bioquímicos en comparación con la hemoglobina reticulocitaria, por tal motivo se cree que esta, no tiene ningún efecto sobre la dinámica del hierro de los reticulocitos ${ }^{(47,56,57)}$, se sugiere sin embargo realizar más estudios al respecto teniendo en cuenta que algunas investigaciones mencionan que aun estos índices eritrocitarios, carecen de suficiente sensibilidad y especificidad para ser utilizados como herramientas de diagnóstico en donantes en riesgo de deficiencia de hierro(56).

\subsection{Determinación de la hemoglobina reticulocitaria}

El $\mathrm{CHr}$ es el producto de la medición de la concentración de la hemoglobina celular y el volumen celular de los reticulocitos, para ello, es importante establecer un volumen celular de referencia en los eritrocitos y en los reticulocitos. Mediante la medición de la dispersión de la luz frontal se establece una señal proporcional al tamaño de los eritrocitos (rbc-y) y una proporcional al tamaño de los reticulocitos (rety) ${ }^{(4,58) .}$

Para calcular el tamaño de los reticulocitos, en términos generales, se induce a que las células adopten una forma esférica y luego se lee la dispersión de la luz en dos ángulos diferentes, uno alto $\left(5^{\circ}\right.$ a $\left.20^{\circ}\right)$, que ofrece información sobre la refracción celular, y otro bajo $\left(2^{\circ}\right.$ a $\left.3^{\circ}\right)$, al proporcionar el volumen celular y a partir de estas dos mediciones, se calcula el volumen celular de los reticulocitos en fentolitros $(4,59)$. 
Partiendo de este punto de referencia y de la tinción del ácido ribonucleico presente en los reticulocitos y ausente en los eritrocitos, se pueden diferenciar ambas poblaciones y calcular el promedio de $\mathrm{CHr}$ en picogramos (pg), teniendo en cuenta el volumen celular de los reticulocitos y el contenido de hemoglobina de cada uno de ellos(60).

$\mathrm{Si}$ bien este es el principio general para la obtención de $\mathrm{CHr}$, el método para su determinación varía según el autoanalizador de hematología y los principios específicos de medición que aplique cada uno de ellos; dadas estas diferencias, el principal interrogante que surgió es si los valores de $\mathrm{CHr}$ son comparables entre las diferentes metodologías; sin embargo, hoy en día se ha determinado una buena correlación entre los valores obtenidos entre diferentes equipos(61,62).

El CHr, al ser un parámetro derivado del volumen celular, se puede ver afectado especialmente por la concentración de la muestra, se ha observado una estabilidad de resultados cuando la muestra se conserva refrigerada (entre 2 y $4{ }^{\circ} \mathrm{C}$ ) por 48 horas(60). De acuerdo con lo reportado en la literatura, los valores de variabilidad biológica para la $\mathrm{CHr}$ se encuentran entre 24,1 pg a 35,8 pg. Cuando se habla de una deficiencia de hierro el valor se encuentra generalmente inferior a 26 pg. Un contenido de $\mathrm{CHr}$ inferior a 29 pg predice deficiencia de hierro en pacientes tratados con agentes estimuladores de la eritropoyesis, un valor de $\mathrm{CHr}$ mayor a $30.6 \mathrm{pg}$ se considera predictivo de respuesta a tratamiento con hierro vía intravenoso en pacientes en diálisis(63-65).

\subsection{Utilidad Clínica}

La determinación de $\mathrm{CHr}$ refleja la síntesis de la hemoglobina en los precursores eritroides y permite la detección de las primeras etapas de la deficiencia de hierro. Este parámetro ha sido identificado como auxiliar en el diagnóstico diferencial de anemias, las principales ventajas de este parámetro es que es más preciso que los marcadores bioquímicos, como la ferritina, hierro sérico, y la saturación de transferrina en la detección de eritropoyesis deficiente en hierro en pacientes con procesos de inflamación o anemia crónica(66-68).

Esta hemoglobina es de particular importancia en la detección precoz de la disminución de los niveles del depósito de hierro, de igual forma en los pacientes con enfermedad renal que reciben eritropoyetina y se emplea como prueba tamiz en la detección y el manejo de la ferropenia en la población general y en los procesos en donde se suprime la hematopoyesis, como la quimioterapia(31,69).

El CHr también ha demostrado ser útil en la detección del doping por eritropoyetina o como un parámetro de control de terapia con eritropoyetina humana recombinante (rHuEPO), utilizada principalmente en pacientes con enfermedad renal crónica(70,71). El incremento de la eritropoyesis inducida por la administración de rHuEPO no puede ser apoyada por la disponibilidad normal de hierro, ya que puede desarrollar una eritropoyesis deteriorada por una disminución de la incorporación de hierro en la hemoglobina(72), llamada deficiencia funcional de hierro, lo que exige un cambio en la dosis de eritropoyetina o la administración intravenosa de los suplementos de hierro(73).

En términos de precisión y disponibilidad de nuevos parámetros de la serie eritroide y el recuento de reticulocitos son clínicamente importantes tanto para la clasificación fisiopatológica de la anemia como para monitorizar la respuesta medular después de intervenciones terapéuticas, además se ha visto la utilidad junto con otros biomarcadores en la identificación de talasemias y variantes de

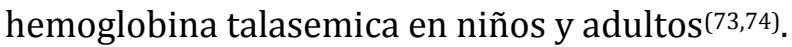

El CHr está incorporado a los autoanalizadores, esto la hace una prueba de fácil acceso, ya que se puede realizar como parte de un examen hematológico habitual. Al estar automatizado, disminuye las posibilidades de error en la interpretación del resultado(47,75); permite obtener el resultado en un tiempo mínimo (el mismo requerido para conocer el resultado de un hemograma) y precisa una muestra de sangre entre 1 y 1,5 ml, colectada en tubo con EDTA. El 
panel clásico de pruebas empleado para diagnosticar el déficit de hierro incluye un hemograma y una bioquímica del metabolismo férrico que consta de la determinación del hierro sérico, transferrina, ferritina, y saturación de transferrina ${ }^{(75) .}$

\section{Conclusiones}

El CHr se establece como un parámetro de gran utilidad en el diagnóstico de anemia ferropénica, debido a que da una idea del hierro que puede ser incorporado a la hemoglobina de los eritrocitos, es un parámetro precoz de detección del déficit de hierro antes de su progresión a anemia por lo que se evidencia un poco antes de los cambios morfológicos generados en los hematíes en la última fase del síndrome anémico.

Según la revisión, se han encontrado otras utilidades del $\mathrm{CHr}$, en pacientes con deficiencia funcional de hierro en estados de inflamación crónica y enfermedad renal crónica, además puede ser utilizado como monitoreo de la terapia con eritropoyetina y la sustitución de hierro en pacientes con insuficiencia renal; se ha empleado como prueba tamiz en la detección y el manejo de la ferropenia en pacientes en procesos en donde se suprime la hematopoyesis como la quimioterapia indicando que es un parámetro de gran importancia clínica, de fácil interpretación, acceso, obtención de los resultados de forma inmediata, mucho más rápido que las pruebas bioquímicas y de menor costo ya que se encuentra incorporado como parámetro en hemogramas automatizados de VI generación.

Según datos de la OMS, los países en desarrollo presentan prevalencia de anemia ferropénica hasta del $41 \%$ para las mujeres en edad fértil, la determinación precoz de esta entidad permitiría instaurar terapias tempranas que eviten las complicaciones de la ferropenia y mejoren la calidad de vida de las pacientes.

En países en vía de desarrollo la implementación de tecnología que permite la realización de parámetros como la $\mathrm{CHr}$ se puede demorar debido a las diversas barreras que afectan la tecnología y la atención en salud.

\section{Conflicto de Interés}

Los autores declaramos que no existe ningún conflicto de interés que ponga en riesgo la validez de los resultados presentados.

\section{Referencias}

1. World Health Organization. The global prevalence of anaemia in 2011 who library cataloguing-in-publication data. the global prevalence of anaemia in (2015th ed.). Geneva: WHO; 2011. Disponible en: apps.who.int/iris/bitstream/10665/177094/1/97892 41564960_eng.pdf

2. Donato H, Cedola A, Rapetti MC, Buys MC, Gutiérrez M, Parias-Nucci R, Schvartzman G. Anemia ferropénica. Guía de diagnóstico y tratamiento. Arch Argent Pediatr. 2010;107(4):353-361. Disponible en: http://www.sap.org.ar/uploads/consensos/anemiaferrop-eacutenica-gu-iacutea-de-diagn-oacutestico-ytratamiento.pdf

3. Urrechaga E, Borque L, Escanero JF. Biomarkers of hypochromia: the contemporary assessment of iron status and erythropoiesis. BioMed Research International, 2013;2013:603786. Disponible en: https://www.ncbi.nlm.nih.gov/pmc/articles/PMC3600 252/

4. Karagülle M, Gündüz E, Sahin-Mutlu F, Olga-Akay M. Clinical significance of reticulocyte hemoglobin content in the diagnosis of iron deficiency anemia. Turkish Journal of Haematology. 2013;30(2):153-156. Disponible https://www.ncbi.nlm.nih.gov/pmc/articles/PMC3878 462/pdf/TJH-30-153.pdf

5. Buttarello M. Laboratory diagnosis of anemia: are the old and new red cell parameters useful in classification and treatment, how?. International Journal of Laboratory Hematology. 2016;38(S1):123-132. Disponible en: http://onlinelibrary.wiley.com/doi/10.1111/ijlh.1250 $0 /$ full

6. Cai J, Wu M, Ren J, Du Y, Long Z, Li G, Yang L. Evaluation of the Efficiency of the Reticulocyte Hemoglobin Content on Diagnosis for Iron Deficiency Anemia in Chinese Adults. Nutrients. 2017;9(5):1-9. Disponible en:https://www.ncbi.nlm.nih.gov/pmc/articles/PMC54 52180/pdf/nutrients-09-00450.pdf

7. Urrechaga-Igartua E, Hoffmann J, Izquierdo-Álvarez S, Escanero JF. Reticulocyte hemoglobin content (MCHr) in the detection of iron deficiency. Journal of Trace Elements in Medicine and Biology. 2017;43:29-32. Disponible https://doi.org/10.1016/J.JTEMB.2016.11.001

8. Hernández-Reyes L, Fundora-Sarraff T, AndradeRuiseco M. El conteo automático de reticulocitos: una herramienta de uso diagnóstico, clínico e investigativo Automated reticulocyte count: a tool for diagnostic, clinical and research use. 2015;31(4):362-371. Disponible en: 
9. Torrens M. Interpretación clínica del hemograma. Revista Clínica Las Condes. 2015;26(6):713-725. Disponible en: https://www.sciencedirect.com/science/article/pii/S0 716864015001480

10. Barragán-Ibañez G, Santoyo-Sánchez A, Ramos-Peñafiel CO. Iron deficiency anaemia. Revista Médica Del Hospital General de México. 2016;79(2):88-97. Disponible desde Internet en: http://linkinghub.elsevier.com/retrieve/pii/S0185106 315000918

11. Rodak-Bernadette F, George A, Fritsma EMK. Hematología Fundamentos y aplicaciones clínicas. España: Ed Panamericana; 2014.

12. Gómez-Casal F. Hematología. España: prensas de la Universidad de Zaragoza; 2016.

13. Moras M, Lefevre SD, Ostuni MA. From Erythroblasts to Mature Red Blood Cells: Organelle Clearance in Mammals. Front Physiol. 2017;8:1076.

14. Iniesta I. Hematopoyesis. España: Ediciones Oblicuas; 2014.

15. Saito $H$, Hayashi $H$. Transformation rate between ferritin and hemosiderin assayed by serum ferritin kinetics in patients with normal iron stores and iron overload. Nagoya Journal of Medical Science. 2015;77(4):571-583. Disponible en: https://www.ncbi.nlm.nih.gov/pmc/articles/PMC4664 589/pdf/2186-3326-77-0571.pdf

16. Saito H. Metabolism of Iron Stores. Nagoya J Med Sci. 2014;76(3-4):235-254. Disponible en: https://www.ncbi.nlm.nih.gov/pmc/articles/PMC4345 694/

17. Alvarez-Argüelles H, Raya-Sánchez JM, GarcíaHernández S, Martín-Santos T, Brito-Barroso ML, Martín-Herrera A, Hernández-Nieto L. Correlación entre los depósitos de hemosiderina en biopsias de médula ósea y marcadores séricos de hierro corporal: validación de un método semicuantitativo de evaluación de la tinción de Perls. Revista Española de Patología. 2012;45(4):218-223.

18. Sociedad Argentina de Hematología. Guías de diagnóstico y tratamiento. Argentina: Sociedad Argentina de Hematología; 2017.

19. Sermini CG, Acevedo MJ, Arredondo M. Biomarcadores del metabolismo y nutrición de hierro. Rev Peru Med Exp Salud Pública. 2017;34(4):690-8. doi: 10.17843/rpmesp.2017.344.3182

20. Crudo C, Erramouspe B, Sueldo E, Arias M. Perls Iron Stain. Hematología. 2016;20(2):243-246. Disponible en: http://www.sah.org.ar/revista/numeros/14\%20vol\% 2020\%20N2\%20\%202016.pdf

21. Manito N, et al. Documento de consenso de la Sociedad Española de Cardiología y la Sociedad Española de Medicina Interna sobre el diagnóstico y tratamiento del déficit de hierro en la insuficiencia cardíaca. Rev Clin Esp. 2016. Disponible en: http://dx.doi.org/10.1016/j.rce.2016.08.001

22. Wolff F, Deleers M, Melot C, Gulbis B, Cotton F. Hepcidin-25: Measurement by LCMS/MS in serum and urine, reference ranges and urinary fractional excretion. Elsevier Inc. 2013;413:99-104.
23. Cailliat MC, Fink NE. Algoritmos de laboratorio para el estudio del estado del hierro. Acta bioquím. clín. latinoam. [Internet]. 2013 ;47(3):507-22. [Citado 2018 Mar 23] Disponible en: http://www.scielo.org.ar/scielo.php?script=sci_arttext \&pid=S0325-29572013000300005

24. Büyükkaragöz $B$, Akgun $N A$, Bulus $A D$, DurmusAydogdu S, Bal C. ¿Puede usarse el receptor soluble de transferrina para diagnosticar anemia ferropénica y evaluar la respuesta al hierro en lactantes con desnutrición aguda moderada?. Arch. argent. pediatr. [Internet]. 2017;115(2):125-132. [Citado 2018 Mar 23] Disponible en:http://dx.doi.org/10.5546/aap.2017.125

25. Eguchi A, Mochizuki T, Tsukada M, Kataoka K, Hamaguchi Y, Oguni S, et al. Clinical Study Serum Hepcidin Levels and Reticulocyte Hemoglobin Concentrations as Indicators of the Iron Status of Peritoneal Dialysis Patients. Int J Nephrol [Internet]. 2012;7:7. [Citado 2018 Enero 22]Disponible en: https://www.hindawi.com/journals/ijn/2012/239476

26. Babaei M, Shafiei S, Bijani A, Heidari B, Hosseyni SR, Vakili-Sadeghi M. Ability of serum ferritin to diagnose iron deficiency anemia in an elderly cohort. Revista Brasileira de Hematologia E Hemoterapia. 2017;39(3):223-228. Disponible en: https://www.ncbi.nlm.nih.gov/pmc/articles/PMC5568 592/pdf/main.pdf

27. Camaschella C, Pagani A. Advances in understanding iron metabolism and its crosstalk with erythropoiesis. $\mathrm{Br} \quad J$ Haematol. 2018;182(4):1-14. doi: 10.1111/bjh.15403

28. Ward DM, Kaplan J. Ferroportin-mediated iron transport: Expression and regulation. Biochimica et Biophysica Acta (BBA) - Molecular Cell Research. 2012;1823(9):1426-1433. Disponible desde Internet en:https://www.ncbi.nlm.nih.gov/pmc/articles/PMC37 18258/pdf/nihms367717.pdf

29. Parodi E, Giraudo MT, Ricceri F, Aurucci ML, Mazzone R, Ramenghi U. Absolute Reticulocyte Count and Reticulocyte Hemoglobin Content as Predictors of Early Response to Exclusive Oral Iron in Children with Iron Deficiency Anemia. Anemia. 2016;2016:1-6. Disponible en:https://www.hindawi.com/journals/anemia/2016/ 7345835/

30. Al-Ghananim RT, Nalbant D, Schmidt RL, Cress GA, Zimmerman MB, Widness JA. Reticulocyte Hemoglobin Content During the First Month of Life in Critically Ill Very Low Birth Weight Neonates Differs From Term Infants, Children, and Adults. Journal of Clinical Laboratory Analysis. 2016;30(4):326-334. Disponible en:

https://www.ncbi.nlm.nih.gov/pmc/articles/PMC4644 110/pdf/nihms680716.pdf

31. Peerschke EIB, Pessin MS, Maslak P. (2014). Using the hemoglobin content of reticulocytes (RET-He) to evaluate anemia in patients with cancer. American Journal of Clinical Pathology. 2014;142(4):506-512. Disponible en: https://www.ncbi.nlm.nih.gov/pmc/articles/PMC4962 332/pdf/nihms802884.pdf 
32. Crudo C, Erramouspe B, Sueldo E, Arias M. Tinción de hierro medular. Coloración de Perls Perls Iron Stain. Hematología. 2016;20(2):243-246. Disponible en: http://www.sah.org.ar/revista/numeros/14\%20vol\% 2020\%20N2\%20\%202016.pdf

33. Daru J, Colman K, Stanworth SJ, De La Salle B, Wood EM, Pasricha SR. (2017). Serum ferritin as an indicator of iron status: what do we need to know? The American Journal of Clinical Nutrition. 2017;106(Suppl 6):1634S1639S.

34. Lichtman, A. (2014). Manual de Hematología. 8 ed. España: McGraw-Hill; 2014.

35. Forrellat-Barrios M, Fernández-Delgado N, HernándezRamírez P. Regulación de la hepcidina y homeostasis del hierro: avances y perspectivas. Revista Cubana de Hematología, Inmunología Y Hemoterapia. 2012;28(4):347-356. Disponible en: http://www.medigraphic.com/pdfs/revcubheminmhe m/rch-2012/rch124d.pdf

36. Piero A, Courtois V, Bastida S, Sánchez-Muniz FJ, Vaquero MP. Deficiencia y sobrecarga de hierro; implicaciones en el estado oxidativo y la salud cardiovascular. Nutrición Hospitalaria. 2010;25(3):350365. Disponible desde Internet en: http://www.redalyc.org/pdf/3092/309226757003.pdf

37. Pfeiffer CM, Looker AC. Laboratory methodologies for indicators of iron status: strengths, limitations, and analytical challenges. The American Journal of Clinical Nutrition. 2017;106(Suppl 6):1606S-1614S.

38. Lynch S, Pfeiffer CM, Georgieff MK, Brittenham G, Fairweather-Tait S, Hurrell RF, et al. Biomarkers of Nutrition for Development (BOND)-Iron Review. J Nutr. 148(suppl_1):1001S-1067S.

39. Northrop-Clewes CA, Thurnham DI. Biomarkers for the differentiation of anemia and their clinical usefulness. Journal of Blood Medicine. 2013;4:11-22. Disponible en: https://www.ncbi.nlm.nih.gov/pmc/articles/PMC3610 441/pdf/jbm-4-011.pdf

40. Quintana-Guzmán EM, Salas-Chaves MdelP. Receptores solubles de transferrina como mejor indicador bioquímico para definir deficiencia de hierro. Acta Bioquímica Clínica Latinoamericana. 2010;44(3):311316.

Disponibleen:http://www.scielo.org.ar/pdf/abcl/v44n 3/v44n3a02.pdf

41. Hoon-Yoon S, Sup-Kim D, Taek-Yu S, Ron-Shin S, YoungChoi D. The usefulness of soluble transferrin receptor in the diagnosis and treatment of iron deficiency anemia in children. Korean J Pediatr. 2015;58(1):15-19. Disponible en: https://www.ncbi.nlm.nih.gov/pmc/articles/PMC4342 776/pdf/kjped-58-15.pdf .

42. Means RT. Pathophysiology in Medicine: Hepcidin and iron regulation in health and disease. Am J Med Sci. 2013;345(1):57-60. Disponible en: https://www.ncbi.nlm.nih.gov/pmc/articles/PMC3430 792/

43. Ganz T. Hepcidin and iron regulation, 10 years later. Blood. 2013;117(17):4425-4433. Disponible en: https://www.ncbi.nlm.nih.gov/pmc/articles/PMC3099 $567 /$
44. Le Petitcorps H, Monti A, Pautas É. Iron deficiency in elderly patients: use of biomarkers. Ann Biol Clin (Paris). 2015;73(6):639-42.

45. Forrellat-Barrios M. Diagnóstico de la deficiencia de hierro: aspectos esenciales. Revista Cubana de Hematología, Inmunología y Hemoterapia. 2017;33(2):1-9.

46. Kemper AR, Fan T, Grossman DC, Phipps MG. Gaps in evidence regarding iron deficiency anemia in pregnant women and young children: summary of US Preventive Services Task Force recommendations. The American journal of clinical nutrition. 2017;106(Supplement 6):1555S-8S.

47. Schapkaitz E. Stability of New Erythrocyte and Reticulocyte Parameters in Testing for Anemia on the Sysmex XN 9000. Laboratory Medicine. 2018;10(4):356-360.

48. Buttarello M, Rauli A, Mezzapelle G. Reticulocyte count and extended reticulocyte parameters by Mindray BC6800: Reference intervals and comparison with Sysmex XE-5000. International Journal of Laboratory Hematology. 2017;39(6):596-603.

49. Mast AE, Blinder MA, Dietzen DJ. Reticulocyte hemoglobin content. American Journal of Hematology. 2008;83(4):307-310.

50. Becker K. Ana. Interpretación del hemograma. Rev. chil. pediatr. [Internet]. 2001;72(5):460-465. [Citado 2018 Mayo 07]; Disponible en: https://scielo.conicyt.cl/scielo.php?script=sci_arttext\& pid=S0370-41062001000500012

51. Torrens Mónica. Interpretación clínica del hemograma. Rev. Med. Clin. Condes [Internet]. 2015;26(6)713-725. [citado 2018 Mayo 07]; Disponible en: https://www.researchgate.net/publication/29000990 0_INTERPRETACION_CLINICA_DEL_HEMOGRAMA

52. Campuzano G. Interpretación del hemograma automatizado: claves para una mejor utilización de la prueba. Med. lab [Internet]. 2013;19(1,2):11-68. [citado 2018 Mayo 07]; Disponible en: http://www.edimeco.com/component/zoo/item/inter pretacion-del-hemograma-automatizado-claves-parauna-mejor-utilizacion-de-la-prueba

53. Brugnara C, Laufer MR, Friedman AJ, Bridges K, Platt $O$. Reticulocyte hemoglobin content (CHr): early indicator of iron deficiency and response to therapy. Blood. 1994;83(10):3100-1.

54. Fishbane S, Galgano C, Langley-Jr RC, Canfield W, Maesaka JK. Reticulocyte hemoglobin content in the evaluation of iron status of hemodialysis patients. Kidney international. 1997;52(1):217-22.

55. Brugnara C, Jo-Hipp M, Irving PJ, Lathrop H, Lee PA, Minchello EM, Winkelman J. Automated reticulocyte counting and measurement of reticulocyte cellular indices: evaluation of the Miles $\mathrm{H}^{*} 3$ blood analyzer. American Journal of Clinical Pathology. 1994;102(5):623-32.

56. Ledesma-Herrera AM, Pedraza-García AL. Hemoglobina reticulocitaria en donantes de sangre. [Trabajo de grado]. Bogotá: Universidad Javeriana; 2010.

57. Eguchi A, Mochizuki T, Tsukada M, Kataoka K, Hamaguchi Y, Oguni S, Tsuchiya K. Clinical Study Serum 
Hepcidin Levels and Reticulocyte Hemoglobin Concentrations as Indicators of the Iron Status of Peritoneal Dialysis Patients. International Journal of Nephrology. 2012;7:7. Disponible en: https://www.hindawi.com/journals/ijn/2012/239476

58. Baart AM, Balvers MGJ, Hopman MTE, Eijsvogels TMH, Klein Gunnewiek JMT, van Kampen CA. Reticulocyte hemoglobin content in a large sample of the general Dutch population and its relation to conventional iron status parameters. Clin Chim Acta. 2018;8(483):20-4.

59. Capone D, Cataldi M, Viniguerra M, Mosca T, Barreta S, Ragosta A, Sorretino A. Reticulocyte Hemoglobin Content Helps Avoid Iron Overload in Hemodialysis Patients: A Retrospective Observational Study. In Vivo. 2017;31(4):709-712. Disponible en: https://www.ncbi.nlm.nih.gov/pmc/articles/PMC5566 927/pdf/in_vivo-31-709.pdf

60. Campuzano G. Hemoglobina Reticulocitaria: Un nuevo parámetro del Hemograma de gran valor en el diagnóstico y manejo de la eritropoyesis deficiente en hierro. Medicina Y Laboratorio. 2015;21(1-2):11-12.

61. Wolf-Grotto HZ. Platelet and reticulocyte new parameters: why and how to use them? Revista Brasileira de Hematologia E Hemoterapia. 20165;38(4):283-284. Disponible en: https://www.ncbi.nlm.nih.gov/pmc/articles/PMC5119 $670 /$ pdf/main.pdf

62. Láser L, Hernández-Reyes H. Avances y aplicación clínica de la citometría hemática automatizada. Revista Cubana Hematología Inmunología Y Hemoterapia. 2013;2929(11):24-3924. Disponible en: http://scielo.sld.cu/pdf/hih/v29n1/hih04113.pdf

63. Canalejo K, Aixalá M, Casella A, Capanera P, Medina J, Jelen A. Evaluación de la fracción de reticulocitos inmaduros como parámetro de ferropenia en el embarazo. Acta Bioquím Clín Latinoam. 2011;45(1):8185. Disponible en: http://www.scielo.org.ar/pdf/abcl/v45n1/v45n1a04.p df

64. Camargo-Morkis IV, Granero-Farias M, Scotti L. Determination of reference ranges for immature platelet and reticulocyte fractions and reticulocyte hemoglobin equivalent. Revista Brasileira de Hematologia E Hemoterapia. 2016;38(4):310-313. Disponible desde Internet en: https://www.sciencedirect.com/science/article/pii/S1 51684841630072X

65. Barbosa-Torino AB, Pererira-Gilberti AdeF, da Costa E, Freire de Lima GA, Zerlotti H. Evaluation of erythrocyte and reticulocyte parameters as indicative of iron deficiency in patients with anemia of chronic disease. Revista Brasileira de Hematologia E Hemoterapia. 2015;3(72):77-81. Disponible desde Internet en: http://www.scielo.br/pdf/rbhh/v37n2/1516-8484rbhh-37-02-00077.pdf

66. Stein J, Dignass AU, Sachsenhausen K. Management of iron deficiency anemia in inflammatory bowel disease a practical approach. Annals of Gastroenterology Ann Gastroenterol. 2013;26(262):104-113. Disponible en: https://www.ncbi.nlm.nih.gov/pmc/articles/PMC3959 949/pdf/AnnGastroenterol-26-104.pdf
67. Nairz M, Theurl I, Wolf D, Weiss G, Nairz M, Theurl I, Wolf D. Main topic Iron deficiency or anemia of inflammation? Differential diagnosis and mechanisms of anemia of inflammation Eisenmangel oder Entzündungsanämie? Wiener Medizinische Wochenschrift. 2016;166:411-423. Disponible en: https://www.ncbi.nlm.nih.gov/pmc/articles/PMC5065 583/pdf/10354_2016_Article_505.pdf

68. Mikhail A, Brown C, Williams JA, Mathrani V, Shrivastava R, Evans J, Bhandari S. Renal association clinical practice guideline on Anaemia of Chronic Kidney Disease. BMC Nephrology. 2017;18:345. Disponible en: https://www.ncbi.nlm.nih.gov/pmc/articles/PMC5709 852/pdf/12882_2017_Article_688.pdf

69. Wang Y, Lu X, He J, Zhao W. Influence of erythropoietin on microvesicles derived from mesenchymal stem cells protecting renal function of chronic kidney disease. Stem Cell Research \& Therapy. 2012;6(1):100. Disponible en: https://www.ncbi.nlm.nih.gov/pmc/articles/PMC4469 245/

70. Davis-Ajami ML, Wu J, Downton K, Ludeman E, Noxon V. Epoetin zeta in the management of anemia associated with chronic kidney disease, differential pharmacology and clinical utility. Biologics: Targets and Therapy. 2014;8:155-167. Disponible en: https://www.ncbi.nlm.nih.gov/pmc/articles/PMC3999 275/pdf/btt-8-155.pdf

71. Wang L, Lijun Di, Noguchi CT. Erythropoietin, a Novel Versatile Player Regulating Energy Metabolism beyond the Erythroid System. Int. J. Biol. Sci. 2014;10(8):921$939 . \quad$ Disponible en: https://www.ncbi.nlm.nih.gov/pmc/articles/PMC4147 225/pdf/ijbsv10p0921.pdf

72. Mairbäurl H, Bogdanova A, Lombardi G. Red blood cells in sports: effects of exercise and training on oxygen supply by red blood cells. Frontiers in Physiology. Front Physiol. 2013;4:332. Disponible en: https://www.ncbi.nlm.nih.gov/pmc/articles/PMC3824 146/pdf/fphys-04-00332.pdf

73. Sudmann ÅA, Piehler A, Urdal P, Shild Å, Sudmann A. (2012). Reticulocyte hemoglobin equivalent to detect thalassemia and thalassemic hemoglobin variants. International Journal of Laboratory Hematology 2012;34:605-613. Disponible en: https://www.ncbi.nlm.nih.gov/pmc/articles/PMC3533 779/pdf/ijlh0034-0605.pdf

74. Buoro S, Mecca T, Seghezzi M, Manenti B, Cerutti L, Dominoni P, Lippi G. Assessment of blood sample stability for complete blood count using the Sysmex XN9000 and Mindray BC-6800 analyzers. Revista Brasileira de Hematologia E Hemoterapia. 2016;38(3):225-239. Disponible desde Internet en:https://www.ncbi.nlm.nih.gov/pmc/articles/PMC49 97904/pdf/main.pdf

75. Ibáñez-Alcalde MM, Vázquez-López MÁ, Ruíz-Sánchez AM, Lendínez-Molinos FJ, Galera-Martínez R, BonilloPerales A, et al. Reference Values of Reticulocyte Hemoglobin Content in Healthy Adolescents. J Pediatr Hematol Oncol. 2018;40(4):298-303. 